Pacific Journal of Mathematic 


\section{COMPLETELY RANDOM MEASURES}

\section{J. F. C. Kingman}

The theory of stochastic processes is concerned with random functions defined on some parameter set. This paper is concerned with the case, which occurs naturally in some practical situations, in which the parameter set is a $\sigma$-algebra of subsets of some space, and the random functions are all measures on this space. Among all such random measures are distinguished some which are called completely random, which have the property that the values they take on disjoint subsets are independent. A representation theorem is proved for all completely random measures satisfying a weak finiteness condition, and as a consequence it is shown that all such measures are necessarily purely atomic.

1. Stochastic processes $X(t)$ whose realisation are nondecreasing functions of a real parameter $t$ occur in a number of applications of probability theory. For instance, the number of events of a point process in the interval $(0, t)$, the 'load function' of a queue input [2], the amount of water entering a reservoir in time $t$, and the local time process in a Markov chain ([8], [7] $\S 14)$, are all processes of this type. In many applications the function $X(t)$ enters as a convenient way of representing a measure on the real line, the Stieltjes measure $\Phi$ defined as the unique Borel measure for which

$$
\Phi(a, b]=X(b+)-X(a+),(-\infty<a<b<\infty) .
$$

For example, in the application to point processes, $\Phi(B)$ is just the number of events occurring in the Borel set $B$. In this and other applications, it would seem in some ways more natural to consider the random measure $\Phi$ rather than the associated function $X$.

The advantage of working directly in terms of random measures becomes more obvious when the space involved is more complicated than the real line. For example, a point process in several dimensions, (as considered by Bartlett [1] and others) would be difficult to work with in terms of the analogue of $X(t)$, but it seems easy and natural to consider a random measure $\Phi$ such that $\Phi(B)$ is the number of points of the process in the set $B$. (Such an approach is implicit in the work of Ryll-Nardzewski [10].) Another application in which it is reasonable to introduce a random measure is one studied by Whittle [11], in which a model for an agricultural situation might be formulated in terms of a measure $\Phi$, with $\Phi(B)$ representing the yield from a plot $B$. 
Returning now to one dimension, one of the simplest stochastic models for the nondecreasing process $X(t)$ is that which assumes that $X(t)$ has independent increments. To this model we can of course apply the extensive theory ([3] Chapter VIII, [9] Chapter XI) of processes with independent, but not necessarily nonnegative, increments. This theory, however, simplifies very considerably when the increments are required to be nonnegative, which suggests that a direct approach might be fruitful.

In terms of the Stieltjes measure $\Phi$ associated with $X$ by (1), the condition that $X$ have independent increments is just the condition that $\Phi$ take independent values on disjoint sets. This latter condition does not involve the special structure of the real line, and enables the whole theory to be extended to more general spaces.

These considerations lead us to introduce the notion of a completely random measure on a general measure space $S$. This is a random measure on $S$ with the property that the values it takes on disjoint sets are independent. A representation theorem is obtained for the possible distributions of $\Phi$, and this is used to prove that a completely random measure can be regarded as the sum of three components, one deterministic, one concentrated on a fixed set of atoms, and one concentrated on a random set of atoms. Thus, except possibly for a deterministic component, a completely random measure is purely atomic. In case $S$ is the real line, this reduces to known results about processes with independent, nonnegative increments.

2. Let $S$ be any set and $\subseteq$ a $\sigma$-algebra of subsets of $S$. To avoid trivial complications, we assume without further comment that

$$
\{x\} \in \subseteq \text { for all } x \in S \text {. }
$$

By a measure on $S$ we shall as usual mean a $\sigma$-additive function from $\subseteq$ into the compactified half-line $R^{+}=[0, \infty]$, taking the value 0 on the empty set $\dot{\phi}$.

We shall be concerned with random measures on $S$, i.e., random variables whose values are measures on $S$. More formally, let $(\Omega, \mathfrak{F}, \boldsymbol{P})$ be a probability space. Then a random measure $\Phi$ defined with respect to this space is a function which associates with each $\omega \in \Omega$ a measure $\Phi_{\omega}$ on $S$ such that, for all $A \in \mathfrak{S}$, the function

$$
\omega \rightarrow \Phi_{\omega}(A)
$$

from $\Omega$ into $R^{+}$is $\mathfrak{F}$-measurable. In what follows, we shall suppress all reference to the underlying probability space $\Omega$, and write $\Phi(A)$ for the random variable $\Phi$. $(A)$.

A random measure $\Phi$ will be said to be completely random if, 
for any finite collection $A_{1}, A_{2}, \cdots, A_{n}$ of disjoint members of $\mathfrak{\varsigma}$, the random variables $\Phi\left(A_{1}\right), \Phi\left(A_{2}\right), \cdots, \Phi\left(A_{n}\right)$ are independent.

Let $\Phi$ be a completely random measure, and let $B_{1}, B_{2}, \cdots, B_{m}$ be members of $\mathfrak{S}$, not necessarily disjoint. Then $\Phi\left(B_{j}\right)$ can, for any $j$, be expressed as the sum of expressions of the form

$$
\Phi\left(B_{1}^{\prime} \cap \mathrm{B}_{2}^{\prime} \cap \cdots \cap B_{m}^{\prime}\right),
$$

where $B_{i}^{\prime}$ is either $B_{i}$ or its complement $S-B_{i}$, and these expressions are, by hypothesis, independent of one another. It follows that the joint distribution of $\Phi\left(B_{j}\right)(j=1,2, \cdots, m)$ is determined once the distribution of $\Phi(A)$ is known for all $A \in \mathfrak{S}$. For this reason we shall concentrate on the distribution of $\Phi(A)$, and the way in which it depends on $A$.

3. For any $t>0$, and any $A \in \mathfrak{S}$, define

$$
\lambda_{t}(A)=-\log \boldsymbol{E}\left\{e^{-t \Phi(A)}\right\},
$$

where $\boldsymbol{E}$ denotes expectation with respect to $\boldsymbol{P}$ and $-\log 0=+\infty$. Then clearly

$$
0 \leqq \lambda_{t}(A) \leqq \infty
$$

and we have

$$
\begin{aligned}
& \lambda_{t}(A)=0 \Leftrightarrow \Phi(A)=0 \text { a.s. }, \\
& \lambda_{t}(A)=\infty \Leftrightarrow \Phi(A)=\infty \text { a.s. }
\end{aligned}
$$

(Here an expression of the form ' $Z$ a.s.' stands for $\boldsymbol{P}\{Z\}=1$.)

If $A_{1}, A_{2}, \cdots$ are countably many disjoint sets in $\mathfrak{S}$, with union $A$, then

$$
\Phi(A)=\sum_{n=1}^{\infty} \Phi\left(A_{n}\right)
$$

and the $\Phi\left(A_{n}\right)$ are independent and nonnegative, so that

$$
\lambda_{t}(A)=\sum_{n=1}^{\infty} \lambda_{t}\left(A_{n}\right)
$$

Hence since $\lambda_{t}\left(\phi^{\prime}\right)=0, \lambda_{t}$ is a measure on $S$. Equation (5) shows that the measures $\lambda_{t}$ (for different values of $t$ ) are mutually absolutely continuous, while (6) shows that they are finite or infinite together. In particular, the ideal $\mathfrak{s}$ of $\mathfrak{S}$ defined by

$$
\mathfrak{g}=\left\{A \in \mathfrak{S} ; \lambda_{t}(A)<\infty\right\}
$$


is independent of $t$, and has the alternative expression

$$
\mathfrak{G}=\{A \in \mathfrak{S} ; \boldsymbol{P}[\Phi(A)<\infty]>0\}
$$

In other words, $A$ belongs to $\mathfrak{Z}$ unless $\Phi(A)$ is almost surely infinite.

The whole discussion simplifies very considerably if we assume that $\lambda_{t}$ is $\sigma$-finite. In view of (6), this amounts to assuming that $\Phi$ satisfies the following condition:

$\mathscr{C}$ : There exists a countable collection $\left\{C_{n}\right\}$ of sets in $\mathfrak{S}$ having union $S$ and such that

$$
\boldsymbol{P}\left\{\Phi\left(C_{n}\right)<\infty\right\}>0 .
$$

Condition $\mathscr{C}$ will be assumed until $\S 11$, where the effect of dropping it will be indicated. We shall take the sets $\left\{C_{n}\right\}$ to be chosen once for all, and we shall arrange the choice, as we clearly may, so that they are disjoint. Certain of the constructions to be made appear to depend on the choice of $\left\{C_{n}\right\}$; the reader will easily verify that this apparent dependence is illusory.

4. Let $\Phi$ be a completely random measure satisfying $\mathscr{C}$, and let the measures $\lambda_{t}$ be as given by (3). Then, since they are mutually absolutely continuous, they have the same set $\mathscr{A}$ of atoms;

$$
\mathscr{A}=\left\{x \in S ; \lambda_{t}(\{x\})>0\right\} .
$$

In fact, (5) shows that

$$
x \in \mathscr{A} \Leftrightarrow \boldsymbol{P}[\Phi(\{x\})>0]>0,
$$

and we therefore call the points of $\mathscr{A}$ the fixed atoms of $\Phi$. Because $\lambda_{t}$ is $\sigma$-finite, the set $\mathscr{A}$ is countable (and hence belongs to $\mathfrak{S}$ ).

If, for any $x \in \mathscr{A}$, we write $\varphi(x)=\Phi(\{x\})$, then $\varphi(x)$ is independent of the values of $\Phi$ on any collection of sets not containing $x$. In particular, the random variables $\varphi(x)(x \in \mathscr{A})$ are independent.

If we write

$$
\Phi_{f}(A)=\Phi(A \cap \mathscr{A}), \Phi_{1}(A)=\Phi(A \cap(S-\mathscr{A})),
$$

then it is clear that each of $\Phi_{f}$ and $\Phi_{1}$ is a completely random measure satisfying $\mathscr{C}$, and that $\Phi=\Phi_{f}+\Phi_{1}$. Moreover, $\Phi_{f}$ and $\Phi_{1}$ are independent, and $\Phi_{f}$ is given by

$$
\Phi_{f}=\sum_{x \in \mathscr{A}} \varphi(x) \delta_{x},
$$

where $\delta_{x}$ is the Dirac measure concentrating mass 1 at the point $x$. Thus we have the following theorem. 
THEOREM 1. Let $\Phi$ be a completely random measure satisfying C. Then

$$
\Phi=\Phi_{f}+\Phi_{1}
$$

where

(i) $\Phi_{f}$ and $\Phi_{1}$ are independent, completely random measures satisfying $\mathscr{C}$,

(ii) $\Phi_{f}$ is given by

$$
\Phi_{f}=\sum_{x \in \mathscr{A}} \varphi(x) \delta_{x},
$$

where $\mathscr{A} \subseteq S$ is countable, and the random variables $\varphi(x)$ are independent, and

(iii) $\Phi_{1}$ has no fixed atoms, so that, for any $x \in S$,

$$
\Phi_{1}(\{x\})=0 \text { a.s., }
$$

Thus from a completely random measure $\Phi$, we can 'subtract off' the fixed atomic component $\Phi_{f}$, and we are left with a completely random measure with no fixed atoms. For this reason we lose nothing by confining attention to completely random measures without fixed atoms, which we now do.

5. Let therefore $\Phi$ be a completely random measure satisfying $\mathscr{C}$ and having no fixed atoms. Then each $\lambda_{t}$ is a nonatomic measure. Let $A \in \mathfrak{B}$, so that $a=\lambda_{1}(A)<\infty$. Since $\lambda_{1}$ is nonatomic, it follows from a well-known result ([5] p. 174; a special case of Lyapunov's theorem) that, for any integer $n$, we can find a measurable dissection $\left\{A_{n j} ; j=1,2, \cdots, n\right\}$ of $A$ for which

$$
\lambda_{1}\left(A_{n j}\right)=a / n \text {. }
$$

Thus

$$
\boldsymbol{E}\left\{e^{-\Phi\left(A_{n}\right)}\right\}=e^{-a / n},
$$

from which, for any $c>0$,

$$
\boldsymbol{P}\left\{\Phi\left(A_{n j}\right) \geqq c\right\} \leqq \frac{1-e^{-a / n}}{1-e^{-c}}
$$

Hence

$$
\lim _{n \rightarrow \infty} \max _{j} \boldsymbol{P}\left\{\Phi\left(A_{n j}\right) \geqq c\right\}=0,
$$

so that the variables $\Phi\left(A_{n j}\right)$ are uniformly asymptotically negligible. But, for each $n$, 


$$
\Phi(A)=\sum_{j=1}^{n} \Phi\left(A_{n j}\right),
$$

and therefore $\Phi(A)$ must be infinitely divisible (or, in the terminology of [9], decomposable).

Since $\Phi(A)$ is nonnegative, it follows from the Lévy-Khinchin representation theorem (in the special form for nonnegative variables given for instance, in [6]) that there exists a finite measure $\Gamma(A,$. on the class $\mathfrak{B}^{+}$of Borel subsets of $R^{+}$, such that, for $t \geqq 0$,

$$
\boldsymbol{E}\left\{e^{-t \phi(\boldsymbol{A})}\right\}=\exp \left[-\int_{R^{+}} k(t, z) \Gamma(A, d z)\right]
$$

where

$$
k(t, z)=\frac{1-e^{-t z}}{1-e^{-z}}, k(t, 0)=t .
$$

In other words, when $A \in \mathfrak{B}$, we must have

$$
\lambda_{t}(A)=\int_{R^{+}} k(t, z) \Gamma(A, d z) .
$$

Our next task is to examine the way in which $\Gamma$ depends on $A$, and to extend (18) to all $A \in \mathfrak{S}$.

6. Let $\mathscr{M}$ denote the class of all (finite or infinite) measures on $\left(R^{+}, \mathfrak{B}^{+}\right)$. If $\mu_{1}, \mu_{2}, \cdots$ belong to $\mathscr{M}$, define a set function $\mu$ by

$$
\mu(E)=\sum_{n=1}^{\infty} \mu_{n}(E),\left(E \in \mathfrak{B}^{+}\right) \text {. }
$$

Then, for any countable dissection of $E$ into Borel sets $E_{m}$, we have

$$
\mu(E)=\sum_{n=1}^{\infty} \sum_{m=1}^{\infty} \mu_{n}\left(E_{m}\right)=\sum_{m=1}^{\infty} \sum_{n=1}^{\infty} \mu_{n}\left(E_{m}\right)=\sum_{m=1}^{\infty} \mu\left(E_{m}\right),
$$

so that $\mu \in \mathscr{M}$. Hence we can define countable sums in $\mathscr{M}$. Moreover, it is immediate from Fubini's theorem and monotone convergence that

$$
\sum_{m} \sum_{n} \mu_{m n}=\sum_{n} \sum_{m} \mu_{m n}
$$

and that, for any nonnegative Borel function $f$,

$$
\int_{R^{+}} f d\left(\sum_{n} \mu_{n}\right)=\sum_{n} \int_{R^{+}} f d \mu_{n} .
$$

Now the function $\Gamma$ which sends $A$ into the measure $\Gamma(A, \cdot)$ defined in the previous section is a function from $\mathfrak{B}$ into $\mathscr{M}$. We show first that $\Gamma$ is $\sigma$-additive, and then that it may be extended from $\vec{z}$ 
to $\mathfrak{S}$ in such a way as to preserve the $\sigma$-additivity.

Let $A_{1}, A_{2}, \cdots$ be disjoint sets in $\mathfrak{S}$ whose union $A$ belongs to $\mathfrak{B}$, so that each $A_{n}$ necessarily belongs to $\mathfrak{B}$. Then, using (18) together with the fact that $\lambda_{t}$ is a measure, we have

$$
\begin{aligned}
\int_{R^{+}} k(t, z) \Gamma(A, d z) & =\sum_{n=1}^{\infty} \int_{R^{+}} k(t, z) \Gamma\left(A_{n}, d z\right) \\
& =\int_{R^{+}} k(t, z) \Gamma^{\prime}(A, d z),
\end{aligned}
$$

where

$$
\Gamma^{\prime}(A, d z)=\sum_{n=1}^{\infty} \Gamma\left(A_{n}, d z\right) .
$$

Putting $t=1$ shows that $\Gamma^{\prime}(A,$.$) is finite. Replacing t$ by $t+1$ and subtracting the results (using the fact that

$$
\left.k(t+1, z)-k(t, z)=e^{-t z}\right),
$$

we get

$$
\int_{R^{+}} e^{-t z} \Gamma(A, d z)=\int_{R^{+}} e^{-t z} \Gamma^{\prime}(A, d z) .
$$

Since this holds for all $t \geqq 0$, it follows that $\Gamma(A,)=.\Gamma^{\prime}(A,$.$) , and$ hence we have proved that, if $\left\{A_{n}\right\}$ is a measurable dissection of $A \in \mathfrak{B}$, then

$$
\Gamma(A, .)=\sum_{n=1}^{\infty} \Gamma\left(A_{n}, .\right) .
$$

For any $A \in \mathfrak{S}$, write

$$
\Gamma_{1}(A, .)=\sum_{n=1}^{\infty} \Gamma\left(A \cap C_{n}, .\right) .
$$

Here $\left\{C_{n}\right\}$ is the dissection occurring in condition $\mathscr{C}$, and the definition has meaning since $A \cap C_{n} \in \mathfrak{g}$. Moreover (21) shows that

$$
\Gamma_{1}(A, .)=\Gamma(A, .)
$$

for $A \in \mathfrak{g}$, so that $\Gamma_{1}$ extends $\Gamma$ from $\mathfrak{g}$ to $\mathfrak{S}$. Again, it follows at once from (19) that $\Gamma_{1}$ is $\sigma$-additive in its first argument Finally, for any $A \in \mathfrak{S}$,

$$
\begin{aligned}
\lambda_{t}(A) & =\sum_{n=1}^{\infty} \lambda_{t}\left(A \cap C_{n}\right) \\
& =\sum_{n=1}^{\infty} \int_{R^{+}} k(t, z) \Gamma\left(A \cap C_{n}, d z\right) \\
& =\int_{R^{+}} k(t, z)\left[\sum_{n=1}^{\infty} \Gamma\left(A \cap C_{n}, d z\right)\right] \\
& =\int_{R^{+}} k(t, z) \Gamma_{1}(A, d z) .
\end{aligned}
$$


Hence $\Gamma_{1}$ is an extension of $\Gamma$ from $\mathfrak{s}$ to $\subseteq$ which preserves the relations (18) and (21). We shall henceforth drop the suffix, and then (18) and (21) are true for all $A \in \mathfrak{S}$. Notice that, because of the definition of sums in $\mathscr{C},(21)$ is equivalent to

$$
\Gamma(A, E)=\sum_{n=1}^{\infty} \Gamma\left(A_{n}, E\right),\left(E \in \mathfrak{B}^{+}\right),
$$

so that, for fixed $E, \Gamma(., E)$ is a measure on $S$.

Summing up these results, we obtain the following theorem.

THEOREM 2. Let $\Phi$ be a completely random measure satisfying $\mathscr{C}$ and having no fixed atoms. Then there exists a function $\Gamma: \mathfrak{S} \times \mathfrak{B}^{+} \rightarrow R^{+}$such that

(i) for $A \in \mathfrak{S}, \Gamma(A,$.$) is a measure on \left(R^{+}, \mathfrak{B}^{+}\right)$

(ii) for $E \in \mathfrak{B}^{+}, \Gamma(., E)$ is a measure on $(S, \subseteq)$,

and (iii) for all $A \in \mathfrak{S}, t>0$,

$$
\boldsymbol{E}\left\{e^{-t \boldsymbol{\phi}(A)}\right\}=\exp \left\{-\int_{R^{+}} \frac{1-e^{-t z}}{1-e^{-z}} \Gamma(A, d z)\right\} .
$$

Remarks. (1) Since, for fixed $t, k(t, z)$ is bounded away from zero and infinity, $\Gamma\left(A, R^{+}\right)<\infty$ if and only if $\lambda_{t}(A)<\infty$, i.e. if and only if $A \in \mathfrak{B}$.

(2) A knowledge of $\Gamma$ determines $\boldsymbol{E}\left\{e^{-t \Phi(A)}\right\}$ for all $t$, and hence determines the finite-dimensional distributions of $\Phi$. Conversely, for a given $\Phi, \Gamma$ is given uniquely by (23).

(3) Since the idea of a function of two arguments which is $\sigma$ additive in each is perhaps unfamiliar, it may be helpful to remark that there is a one-to-one correspondence between the class of functions $\Gamma$ satisfying conditions (i) and (ii) of the theorem and the measures $\Gamma^{*}$ on the product space $S \times R^{+}$, given by

$$
\Gamma^{*}(A \times E)=\Gamma(A, E),\left(A \in \mathfrak{S}, E \in \mathfrak{B}^{+}\right) .
$$

Thus the conclusion of the theorem can be stated:

There exists a measure $\Gamma^{*}$ on $S \times R^{+}$such that

$$
\boldsymbol{E}\left\{e^{-t \Phi(A)}\right\}=\exp \left\{-\int_{R^{+}} k(t, z) \Gamma^{*}(A \times d z)\right\} .
$$

7. We now go on to study in more detail the function $\Gamma$ which determines (via (23)) the finite-dimensional distributions of the completely random measure $\Phi$. We first write

$$
\beta(A)=\Gamma(A,\{0\})
$$


$\beta$ is then a $\sigma$-finite measure on $S$. Next, for any positive integer $v$, we set $I_{v}=(1 / v, 1 / v-1)$, and write

$$
\Delta_{v}(A, E)=\int_{I_{v} \cap E}\left(1-e^{-z}\right)^{-1} \Gamma(A, d z) .
$$

Then $\Delta_{v}$ is a function from $\mathfrak{S} \times \mathfrak{B}^{+}$into $R^{+}, \sigma$-additive in each argument, and (since $\left(1-e^{-z}\right)^{-1}$ is bounded on $\left.I_{v}\right) \Delta_{v}\left(A, R^{+}\right)<\infty$ for $A \in \mathfrak{g}$. Moreover,

$$
\lambda_{t}(A)=t \beta(A)+\sum_{v=1}^{\infty} \int_{R^{+}}\left(1-e^{-t z}\right) \Delta_{v}(A, d z) .
$$

For any $z \in R^{+}$, put

$$
\pi_{v}(A, z)=\Delta_{v}(A,[0, z]), \pi_{v}(A)=\Delta_{v}\left(A, R^{+}\right) .
$$

Then $\pi_{v}(., z)$ and $\pi_{v}($.$) are measures on S$ which are finite on $\mathfrak{g}$, and are thus $\sigma$-finite (by $\mathscr{C}$ ), and moreover $\pi_{v}(., z)$ is absolutely continuous with respect to $\pi_{v}($.$) . For any z$ in the set $Q^{+}$of finite rationals in $R^{+}$let $F_{v}(., z)$ be (a version of) the Radon-Nikodym derivative of $\pi_{v}(., z)$ with respect to $\pi_{v}($.$) , so that$

$$
\pi_{v}(A, z)=\int_{A} F_{v}(x, z) \pi_{v}(d x),\left(A \in \mathfrak{S}, z \in Q^{+}\right) .
$$

If $z_{1}<z_{2}$ are rational, then $\pi_{v}\left(., z_{1}\right) \leqq \pi_{v}\left(., z_{2}\right)$, so that

$$
F_{v}\left(x, z_{1}\right) \leqq F_{v}\left(x, z_{2}\right)
$$

for almost all $x$ (modulo $\pi_{v}$ ). Hence (since $Q^{+}$is countable) for $\pi_{v}$. almost all $x, F_{v}(x,$.$) is a nondecreasing function of z \in Q^{+}$. For any $z \in Q^{+}$,

$$
\begin{aligned}
\pi_{v}(A, z) & =\lim _{n \rightarrow \infty} \pi_{v}\left(A, z+n^{-1}\right) \\
& =\lim _{n \rightarrow \infty} \int_{A} F_{v}\left(x, z+n^{-1}\right) \pi_{v}(d x) \\
& =\int_{A} F_{v}(x, z+) \pi_{v}(d x),
\end{aligned}
$$

so that, for $\pi_{v}$-almost all $x, F_{v}(x, z+)=F_{v}(x, z)$. A similar argument shows that, for $\pi_{v}$-almost all $x$,

$$
\lim _{n \rightarrow \infty} F_{v}(x, n)=1 \text {. }
$$

Hence, by changing $F_{v}(x,$.$) on a \pi_{v}$-null set of values of $x$, we can find a function $F_{v}: S \times Q^{+} \rightarrow R$ such that, for each $x \in S, F_{v}(x,$.$) is$ nondecreasing and right-continuous, with 


$$
F_{v}(x, 0)=0, \lim _{z \rightarrow \infty} F_{v}(x, z)=1,
$$

and such that (30) holds.

This function defines, for each $x$, a probability measure $p_{v}(x,$. on $\left(R^{+}, \mathfrak{B}^{+}\right)$by

$$
p_{v}\left(x,\left(z_{1}, z_{2}\right]\right)=F_{v}\left(x, z_{2}\right)-F_{v}\left(x, z_{1}\right),\left(z_{1}<z_{2} \in Q^{+}\right),
$$

which satisfies

$$
\pi_{v}(A, z)=\int_{\Delta} p_{v}(x,(0, z]) \pi_{v}(d x),\left(z \in Q^{+}\right) .
$$

Hence, by (29),

$$
\Delta_{v}(A, E)=\int_{A} \int_{E} p_{v}(x, d z) \pi_{v}(d x),\left(A \in \mathfrak{S}, E \in \mathfrak{B}^{+}\right) .
$$

Moreover, it is clear from the way in which $p_{v}$ has been constructed that $p_{v}(x, E)$ is a measurable function of $x$, and that

$$
p_{v}\left(x, I_{v}\right)=1 \text {. }
$$

Combining (28) and (33), we have

$$
\begin{aligned}
\lambda_{t}(A) & =t \beta(A)+\sum_{v=1}^{\infty} \int_{\Lambda} \int_{R^{+}}\left(1-e^{-t z}\right) p_{v}(x, d z) \pi_{v}(d x) \\
& =t \beta(A)+\sum_{v=1}^{\infty} \int_{A} \pi_{v}(d x)\left[1-\int_{R^{+}} e^{-t z} p_{v}(x, d z)\right] .
\end{aligned}
$$

Hence Theorem 2 leads to the following result:

THEOREM 3. Let $\Phi$ be a completely random measure satisfying $\mathscr{C}$ and having no fixed atoms. Then there exist $\sigma$-finite measures $\beta, \pi_{1}, \pi_{2}, \cdots$ on $S$, and probability measures

$$
p_{v}(x, .)(v=1,2, \cdots ; x \in S)
$$

on $R^{+}$with $p_{v}(., E)$ measurable for each $E \in \mathfrak{B}^{+}$, such that, for all $t>0$,

$$
\boldsymbol{E}\left\{e^{-t \Phi(\boldsymbol{A})}\right\}=\exp \left\{-t \beta(A)-\sum_{v=1}^{\infty} \int_{\boldsymbol{A}}\left[1-p_{v}^{*}(x, t)\right] \pi_{v}(d x)\right\}
$$

where

$$
p_{v}^{*}(x, t)=\int_{R^{+}} e^{-t z} p_{v}(x, d z)
$$

REMARK. It may seem that we can say more about the measures 
$\beta, \pi_{1}, \pi_{2}, \cdots$ than that they are $\sigma$-finite, because a countable dissection of $S$ can be chosen on which all the measures are simultaneously finite. Call a collection $\mathscr{K}$ of measures on $S$ uniformly $\sigma$-finite if there exists a countable dissection $\left\{A_{n}\right\}$ of $S$ with $\mu\left(A_{n}\right)<\infty$ for all $n$ and all $\mu \in \mathscr{K}$. Then $\left\{\beta, \pi_{1}, \pi_{2}, \cdots\right\}$ is a uniformly $\sigma$-finite collection of measures. However, this strengthening is only apparent, since any countable collection of $\sigma$-finite measures is uniformly $\sigma$-finite. This result is proved in Appendix 1.

8. If we combine Theorems 1 and 3 , we see that the finite-dimensional distributions of a completely random measure $\Phi$ satisfying $\mathscr{C}$ are completely described by the following characteristics :

(i) a countable subset $\mathscr{A}$ of $S$ (the set of fixed atoms),

(ii) for every $x \in \mathscr{A}$, a probability measure $q(x,$.$) on R^{+}$(the distribution of $\left.\varphi^{\prime}(x)\right)$,

(iii) a nonatomic $\sigma$-finite measure $\beta$ on $S$,

(iv) for each positive integer $v$, a nonatomic $\sigma$-finite measure $\pi_{v}$ on $S$,

(v) for each positive integer $v$, and every $x \in S$, a probability measure $p_{v}(x,$.$) on R^{+}-\{0\}$, with $p_{v}(., E)$ measurable for each $E \in \mathfrak{B}^{+}$.

In terms of these characteristics, the distribution of $\Phi(A)$ is given by

$$
\begin{aligned}
& \log \boldsymbol{E}\left[e^{-t \Phi(\Delta)}\right\}= \\
& \sum_{x \in \mathscr{\Re} \cap A} \log q^{*}(x, t)-t \beta(A)-\sum_{v=1}^{\infty} \int_{A}\left[1-p^{*}(x, t)\right] \pi_{v}(d x),
\end{aligned}
$$

where

$$
q^{*}(x, t)=\int_{R^{+}} e^{-t z} q(x, d z)
$$

We now prove a result which is a converse to the theorems already proved.

THEOREM 4. If $\mathscr{A}, q, \beta, \pi_{v}, p_{v}$ are given satisfying (i)-(v), then there exists a completely random measure $\Phi$ satisfying $\mathscr{C}$ and having distributions given by (37).

We prove the theorem by constructing completely random measures $\Phi_{f}, \Phi_{d}, \Phi_{1}, \Phi_{2}, \Phi_{3}, \cdots$ such that

$$
\begin{aligned}
& \boldsymbol{E}\left\{e^{-t \Phi_{f}(A)}\right\}=\prod_{x \in \mathscr{A} \cap \boldsymbol{A}} q^{*}(x, t), \\
& \boldsymbol{E}\left\{e^{-t \Phi_{d}(A)}\right\}=e^{-t \beta(A)},
\end{aligned}
$$

and 


$$
\left.\boldsymbol{E}\left\{e^{-t \Phi_{v}(\Delta)}\right\}=\exp \left\{-\int_{\Delta}\left[1-p_{v}^{*}(x, t)\right] \pi_{v}(d x)\right\}, \quad v=1,2, \cdots\right) .
$$

If all these measures are taken to be independent, and if

$$
\Phi_{0}=\sum_{v=1}^{\infty} \Phi_{v}
$$

then

$$
\Phi=\Phi_{f}+\Phi_{d}+\Phi_{0}
$$

is a completely random measure satisfying (37). Moreover, it is easily verified (using the result of Appendix 1) that $\Phi$ satisfies $\mathscr{C}$.

The first component $\Phi_{f}$ is obtained by taking independent random variables $\varphi(x)(x \in \mathscr{A})$ such that $\varphi(x)$ has probability measure $q(x,$.$) ,$ and setting

$$
\Phi_{f}=\sum \varphi(x) \delta_{x} \text {. }
$$

Then $\Phi_{f}$ is a completely random measure satisfying (39). It is clearly concentrated on the countable set $\mathscr{A}$.

The second component is constructed trivially by putting

$$
\Phi_{d}=\beta
$$

so that $\Phi_{d}$ is purely deterministic.

To obtain $\Phi_{v}$ (where $v$ is a positive integer), first construct a Poisson process $\Pi_{v}$ on $S$ according to the measure $\pi_{v}$ (see Appendix 2). Thus $\Pi_{v}$ will consist of a countable number of random points of $S$, the number of points in disjoint sets being independent, the number in a set $A$ having a Poisson distribution with mean $\pi_{v}(A)$ if this is finite, and otherwise being almost surely infinite. Conditional on $\Pi_{v}$, construct, for each $x \in \Pi_{v}$, a random variable $\varphi(x)$ having distribution $p_{v}(x,$.$) , different \varphi(x)$ being independent. Then elementary computations show that

$$
\Phi_{v}=\sum_{x \in \Pi_{v}} \varphi(x) \delta_{x}
$$

is a completely random measure satisfying (41).

Thus the proof of the theorem is complete.

If we call two random measures equivalent if they have the same finite-dimensional distributions, then we have proved that any completely random measure satisfying $\mathscr{C}$ is equivalent to one of the form implied by equations (42)-(46). In particular we have the following result.

THEOREM 5. Every completely random measure satisfying $\mathscr{C}$ is 
equivalent to the sum of two such measures, one of which is purely deterministic and the other purely atomic.

9. If $\Phi$ is any completely random measure, then it is an immediate consequence of the monotone convergence theorem that the function $m$ given by

$$
m(A)=\boldsymbol{E}\{\Phi(A)\}, \quad(A \in \mathfrak{S}),
$$

is a measure on $S$. If $\Phi$ satisfies $\mathscr{C}$, and thus has a distribution given by (37), then

$$
m(A)=\sum_{2 \cap_{A} A} \int_{R^{+}} z q(x, d z)+\beta(A)+\sum_{v=1}^{\infty} \int_{A} \pi_{v}(d x) \int_{R^{+}} p_{v}(x, d z) .
$$

Notice that, even under $\mathscr{C}, m$ need not be $\sigma$-finite.

A rather more complicated set function is that given by

$$
V(A)=\operatorname{var}\{\Phi(A)\},(A \in S) .
$$

Here var $(X)$ denotes the variance of $X$, with $\operatorname{var}(X)=\infty$ if $\boldsymbol{E}\left(X^{2}\right)=\infty$. If $A$ and $B$ are disjoint, the independence of $\Phi(A)$ and $\Phi(B)$ shows that

$$
V(A \cup B)=V(A)+V(B),
$$

so that $V$ is a finitely additive, but not necessarily $\sigma$-additive, set function on $S$. However, it is easy to show that, if $V(A)<\infty$, then the restriction of $V$ to subsets of $A$ is $\sigma$-additive. For a discussion of $V$ when $S=R^{2}$ we refer to [11].

There are a number of other set functions connected with $\Phi$ which are of some interest. In particular, we leave it to the reader to verify that

$$
\lambda_{\infty}(A)=-\log \boldsymbol{P}\{\Phi(A)=0\}, \quad(A \in \mathfrak{S})
$$

is a measure on $S$, while

$$
\lambda_{0}(A)=-\log \boldsymbol{P}\{\Phi(A)=\infty\}, \quad(A \in \mathfrak{S})
$$

defines a finitely additive, but not in general $\sigma$-additive, set function on $S$.

10. In the decomposition (43) of a completely random measure satisfying $\mathscr{C}$, the fixed atomic component $\Phi_{f}$ and the deterministic component $\Phi_{d}$ are relatively uninteresting, and we therefore concentrate attention on the third component $\Phi_{0}$, which we call the ordinary component of $\Phi$. This is a purely atomic measure, and its atoms form the superposition 


$$
\Pi=\bigcup_{v=1}^{\infty} \Pi_{v}
$$

of the independent Poisson processes $\Pi_{v}$. Notice that, since the measures $\pi_{v}$ are nonatomic, the sets $\Pi_{v}$ are almost certainly disjoint.

Because a superposition of independent Poisson processes is itself a Poisson process, it follows that the atoms of $\Phi_{0}$ follow a Poisson process corresponding to the measure

$$
\pi=\sum_{v=1}^{\infty} \pi_{v}
$$

This last measure is not necessarily $\sigma$-finite. It is this fact that necessitates the awkward splitting of $\Phi_{0}$ into its components

$$
\Phi_{1}, \Phi_{2}, \cdots \text {. }
$$

11. All the above analysis has been carried out using the condition $\mathscr{C}$ and it is worthwhile noting the points at which this condition enters the argument. If it is not assumed, the measures $\lambda_{t}$ can still be defined by (3), but they no longer need be $\sigma$-finite. Thus, if $\sigma_{\mathfrak{s}}$ is the $\sigma$-ideal generated by $\mathfrak{g}$ (the class of countable unions of sets in $\mathfrak{S}), \sigma \mathfrak{S}$ may be a proper sub- $\sigma$-ideal of $\mathfrak{S}$.

The first application of $\mathscr{C}$ occurs in $\S 4$, where it is used to establish the countability of the set $\mathscr{A}$ of fixed atoms. If $\mathscr{C}$ is not assumed, $\mathscr{A}$ may be uncountable, and indeed nonmeasurable. When this is so, there is no obvious way of 'splitting off' the fixed atomic component as is done in Theorem 1.

The analysis of Theorem 2 goes through, as long as we restrict attention to sets in $\sigma \mathfrak{s}$. Thus $\Gamma$ is defined for $A \in \mathfrak{S}$, and then extended as in $\S 6$ to $\sigma$ s. If we then set

$$
\Gamma(A, E)=\infty,(A \in \sigma \mathfrak{s}, E \neq \phi),
$$

(23) is valid for all $A \in \mathbb{S}$.

The argument of $\S 7$, in which the structure of $\Gamma$ is examined in detail, relies heavily on the Radon-Nikodym theorem, and so requires some sort of $\sigma$-finiteness restriction. The condition $\mathscr{C}$ seems to be the simplest such restriction. Whether Theorem 5 is true if $\mathscr{C}$ is not assumed I do not know.

Since $\mathscr{C}$ is, in a sense, a condition of $\sigma$-finiteness on the completely random measure $\Phi$, it is worth asking what relation it bears to the more obvious condition,

$$
\mathscr{C}^{\prime}: \Phi_{\omega} \text { is } \sigma \text {-finite for almost all } \omega \in \Omega \text {. }
$$

The trivial example in which $S$ has just one point $s$ and 


$$
0<\boldsymbol{P}\{\Phi(\{s\})=\infty\}<1
$$

shows that $\mathscr{C}$ does not imply $\mathscr{C}^{\prime}$. It is also true that $\mathscr{C}^{\prime}$ does not imply $\mathscr{C}$; in fact it is possible to construct a completely random measure $\Phi$ on the Borel subsets of $R$ with the following properties ( $m$ denoting Lebesgue measure):

(i) $\Phi_{\omega}$ is $\sigma$-finite for all $\omega$,

(ii) if $m(A)>0$, then $\Phi(A)=\infty$ a.s.,

(iii) if $m(A)=0$, then $\Phi(A)=0$ a.s.

In this case $\mathfrak{Z}$ (and so also $\sigma \mathfrak{G}$ ) consists of the sets of Lebesgue measure zero and so $\mathscr{C}$ is not satisfied. The details of the construction are given in Appendix 3; the result shows the depth of pathology which can be reached if $\mathscr{C}$ is not assumed.

Appendix 1. Uniform $\sigma$-finiteness. The concept of a uniformly $\sigma$-finite collection of measures was defined in $\S 7$. It is trivial that the members of such a collection must each be $\sigma$-finite, and that a finite collection of $\sigma$-finite measures is uniformly $\sigma$-finite. Less trivial is the corresponding result for countable collections, which is a corollary of the following theorem, which is itself related to a result of Feldman ([4], Theorem 7.4).

THeOREM 6. If $\mu_{1}, \mu_{2}, \cdots$ are $\sigma$-finite measures on a space $(S, \mathfrak{S})$, there exist strictly positive numbers $c_{1}, c_{2}, \cdots$ such that $\sum_{n=1}^{\infty} c_{n} \mu_{n}$ is $\sigma$-finite.

Proof. Since $\mu_{n}$ is $\sigma$-finite, it is dominated by a probability measure $\lambda_{n}$, and so by the probability measure

$$
\lambda=\sum_{n=1}^{\infty} 2^{-n} \lambda_{n}
$$

Hence, for some finite nonnegative function $f_{n}$,

$$
\mu_{n}(d x)=f_{n}(x) \lambda(d x) .
$$

As $a \rightarrow \infty, \lambda\left\{x ; f_{n}(x)>a\right\} \rightarrow 0$, and hence we can choose $a_{n}$ so that, if $A=\left\{x ; f_{n}(x)>a_{n}\right\}$, then $\lambda\left(A_{n}\right)<2^{-n}$. We now write

$$
c_{n}=2^{-n} a_{n}^{-1}, \mu=\sum_{n=1}^{\infty} c_{n} \mu_{n} ;
$$

it suffices to prove that $\mu$ is $\sigma$-finite.

If

$$
B_{n}=\bigcup_{k=n+1}^{\infty} A_{k} \quad(n=0,1,2, \cdots),
$$


then

$$
\lambda\left(B_{n}\right) \leqq \sum_{k=n+1}^{\infty} \lambda\left(A_{k}\right)<\sum_{k=n+1}^{\infty} 2^{-k}=2^{-n},
$$

and therefore, if

$$
B_{\infty}=\bigcap_{n=0}^{\infty} B_{n}
$$

we have $\lambda\left(B_{\infty}\right)=0$.

Hence $\mu_{n}\left(B_{\infty}\right)=0$ for all $n$, and so $\mu\left(B_{\infty}\right)=0$, and it suffices to prove that $\mu$ is $\sigma$-finite on $S-B_{\infty}$. Now

$$
S-B_{\infty}=\bigcup_{n=0}^{\infty}\left(A_{n}-B_{n}\right)
$$

$\left(A_{0}=S\right)$ and it thus suffices to prove that $\mu$ is $\sigma$-finite on $A_{n}-B_{n}$. To do this, write

$$
\mu=c_{1} \mu_{1}+c_{2} \mu_{2}+\cdots+c_{n} \mu_{n}+\sum_{r=n+1}^{\infty} c_{r} \mu_{r} .
$$

On the right hand side the first $n$ terms are $\sigma$-finite on $A_{n}-B_{n}$, and it suffices to show that the last is also $\sigma$-finite. It is in fact totally fiaite on $A_{n}-B_{n}$, since

$$
\begin{aligned}
\left(\sum_{r=n+1}^{\infty} c_{r} \mu_{r}\right)\left(A_{n}-B_{n}\right) & =\sum_{r=n+1}^{\infty} c_{r} \int_{\Delta_{n}-B_{n}} f_{r}(x) \lambda(d x) \\
& \leqq \sum_{r=n+1}^{\infty} c_{r} \int_{\Lambda_{n}-B_{n}} a_{r} \lambda(d x) \\
& \leqq \sum_{r=n+1}^{\infty} c_{r} a_{r}=2^{-n}<\infty .
\end{aligned}
$$

This completes the proof.

COROLlary. There exists a countable dissection $\left\{A_{m}\right\}$ of $S$ with $\mu_{n}\left(A_{m}\right)<\infty$ for all $m, n$.

Proof. Take a dissection $\left\{A_{m}\right\}$ with $\mu\left(A_{m}\right)<\infty$. Then

$$
\lambda_{n}\left(A_{m}\right) \leqq c_{n}^{-1} \mu\left(A_{m}\right)<\infty .
$$

Appendix 2. The construction of Poisson processes. By a Poisson process on an abstract space $S$ we mean a random set of points with the property that the numbers of points in disjoint sets are independent Poisson variates. More precisely, let $(S, \mathfrak{S}, \mu)$ be a measure space, let $\Pi$ be a random subset of $S$ and let

$$
N(A)=\#(A \cap \Pi),(A \in \mathfrak{S}) .
$$


(Here, for any set $B, \# B$ denotes the number of points in $B$ if $B$ is finite, and $+\infty$ if $B$ is infinite.) Then we say that $\Pi$ is a Poisson process with measure $\mu$ if

(i) whenever $A_{1}, A_{2}, \cdots, A_{n}$ are disjoint numbers of $\mathfrak{S}$,

$$
N\left(A_{1}\right) N,\left(A_{2}\right), \cdots, N\left(A_{n}\right)
$$

are independent,

(ii) if $\mu(A)<\infty$, then $N(A)$ has a Poisson distribution with mean $\mu(A)$,

(iii) if $\mu(A)=\infty$, then $N(A)=\infty$ a.s.

Thus, in the terminology of this paper, $I$ is a Poisson process with measure $\mu$ if and only if $N$ is a completely random measure with (in the notation of $\S 3$ )

$$
\lambda_{t}(A)=\mu(A)\left(1-e^{-t}\right) .
$$

It is far from obvious that, given a space $(S, \mathcal{S}, \mu)$, there does exist a Poisson process as described above. The object of this appendix, which is the outcome of discussions with Professor D. G. Kendall, is to show how, under suitable conditions, such a process can be constructed.

We first remark that, for a Poisson process to exist, $\mu$ must be nonatomic. For, if $x \in S$ has $\mu\{x\}=a>0$, we have

$$
\boldsymbol{P}\{N(\{x\}) \leqq 1\}=e^{-a}+a e^{-a}<1,
$$

which contradicts the obvious fact that

$$
N(\{x\})=\#(\{x\} \cap \Pi) \leqq 1 .
$$

It is, of course, possible to modify our definition to avoid this difficulty, but this seems hardly worthwhile, and we shall be content to assume that $\mu$ is nonatomic.

The obvious way to proceed to the construction of $\Pi$ is as follows. As in $\S 2$, the assumption that $N$ is a completely random measure satisfying (56) leads to a consistent family of finite-dimensional distributions for the stochastic process

$$
\{N(A) ; A \in \mathfrak{S}\} \text {. }
$$

Hence, by the Daniell-Kolmogorov theorem, there does exist a stochastic process with these finite-dimensional distributions. In particular, $N$ is almost surely a finitely-additive, integer-valued set function satisfying (i)-(iii). The theorem is not, however, sufficiently strong to ensure that $N$ is $\sigma$-additive. Kendall (private communication) has shown how this sort of difficulty may be avoided by powerful general methods, but in the simple case of a Poisson process it is possible to proceed 
in a more elementary way. To do this we need to make a restriction on the measure $\mu$ (apart from nonatomicity), and the result is given in the following theorem.

Theorem 7. Let $(S, \mathcal{S}, \mu)$ be a nonatomic measure space, and suppose that $\mu$ can be expressed as the sum of a countable number of $\sigma$-finite measures. Then there exists a Poisson process $I I$ on $S$ with measure $\mu$ and $\Pi$ is almost certainly countable.

Proof. Consider first the case in which $\mu$ is totally finite, and write $a=\mu(S)$. Choose independent random variables $X_{1}, X_{2}, \cdots, N$, where

(i) $X_{n}$ takes values in $S$, and has distribution given by the measure $a^{-1} \mu$, and

(ii) $N$ has a Poisson distribution with mean $a$. Write

$$
\Pi=\left\{X_{1}, X_{2}, \cdots, X_{N}\right\} .
$$

Then elementary (if tedious) computations show that $I$ is a Poisson process with measure $\mu$. Clearly $\Pi$ is almost certainly finite.

Now suppose that $\mu$ is $\sigma$-finite. Then we can find finite measures $\mu_{1}, \mu_{2}, \cdots$ such that $\mu=\Sigma \mu_{j}$. Construct, as in the previous paragraph Poisson processes $\Pi_{j}$ on $\left(S, \mathcal{S}, \mu_{j}\right)$, and do this so that the $\Pi_{j}$ are independent. Then easily

$$
\Pi=\bigcup_{j} \Pi_{j}
$$

is a Poisson process with measure $\mu$, and is almost surely countable. An exactly similar argument extends the result to countable sums of $\sigma$-finite measures, and preserves the countability of $\Pi$. Hence the theorem is proved.

The Poisson processes discussed in $\S 8$ all correspond to measures which satisfy the conditions of Theorem 7, and hence the argument of that section is completed.

It is perhaps worth stressing that the Poisson process $I I$ can be countable even when the underlying measure $\mu$ is not $\sigma$-finite.

Appendix 3. A pathological example. The object of this appendix is to give details of the completely random measure referred to at the end of $\S 11$. This was a measure $\Phi=\Phi$. On $(R, \subseteq)$ with

(i) $\Phi_{\omega} \sigma$-finite for-all $\omega$,

(ii) $\Phi(A)=\infty$ a.s. whenever $A$ has positive Lebesgue measure $m(A)$,

(iii) $\Phi(A)=0$ a.s. whenever $A$ has zero Lebesgue measure. 
Because the distribution of $\Phi(A)$ is degenerate, this random measure is in fact completely random.

Take the probability space $\Omega$ to be the unit interval, and $\boldsymbol{P}$ to be Lebesgue measure on its Lebesgue subsets. Then define a random measure $\Phi$ on $(R, \mathfrak{B})$ by

$$
\Phi_{\omega}(A)=\#[A \cap(\omega+Q)],(A \in \mathfrak{B}) .
$$

In other words, $\Phi_{\omega}$ is counting measure on the translate $(\omega+Q)$ of the rationals, and is thus $\sigma$-finite.

If $\chi_{A}$ is the characteristic function of $A$, then

$$
\Phi_{\omega}(A)=\sum_{r \in Q} \quad \chi_{A}(\omega+r) .
$$

showing that $\Phi_{\omega}(A)$ is a measurable function of $\omega$. If $m(A)=0$,

$$
\chi_{\Delta}(\omega+r)=0
$$

for almost all $\omega$, and so $\Phi(A)=0$ a.s. Hence $\Phi$ is a random measure satisfying (i) and (iii), and it remains only to prove (ii).

Thus let $A$ have $m(A)>0$. Then there exists $r \in Q$ with

$$
m\{A \cap(r, r+1)\}>0,
$$

and, from (58), for any integers $n, q$,

$$
\Phi_{\omega}(A) \geqq \sum_{k=1}^{n} \chi_{A}(\omega+r+k / q) \text {. }
$$

As $q \rightarrow \infty$, the right hand side converges in $L^{1}(\Omega)$, and hence in measure, to

$$
\sum_{k=1}^{n} \chi_{A}(\omega+r)=n \chi_{A}(\omega+r) .
$$

Hence

$$
\begin{aligned}
\boldsymbol{P}\left\{\Phi_{\omega}(A) \geqq n\right\} & \geqq \boldsymbol{P}\left\{\sum_{k=1}^{n} \chi_{\Delta}(\omega+r+k / q) \geqq n\right\} \\
& \rightarrow \boldsymbol{P}\left\{n \chi_{\Delta}(\omega+r) \geqq n\right\} \\
& =\boldsymbol{P}\{\omega+r \in A\} \\
& =\boldsymbol{P}\{\omega \in A-r\} \\
& =m\{(0,1) \cap(A-r)\} \\
& =m\{A \cap(r, r+1)\},
\end{aligned}
$$

and letting $n \rightarrow \infty$

$$
\boldsymbol{P}\left\{\Phi_{\omega}(A)=\infty\right\} \geqq m\{A \cap(r, r+1)\}>0 .
$$


Now let $E$ be the set of $\omega$ for which $\Phi_{\omega}(A)=\infty$, so that $m(E)>0$. From the definition of $\Phi$ it is clear that

$$
\omega \in E, r \in Q, \omega+r \in(0,1) \Rightarrow \omega+r \in E .
$$

Hence, if $G(x)=m\{E \cap(0, x]\},(0<x<1)$, we have

$$
G(x+r)-G(r)=G(x),(0<x<x+r \lessdot 1, r \in Q) .
$$

Since $G$ is right-continuous,

$$
G(x+y)=G(x)+G(y)
$$

for all $x, y>0, x+y<1$, and hence

$$
G(x)=x G(1)=x m(E) \text {. }
$$

Thus, for almost all $x \in(0,1)$,

$$
\chi_{E}(x)=G^{\prime}(x)=m(E),
$$

and since $\chi_{E}=0$ or 1 and $m(E)>0$ it follows that $m(E)=1$. Thus

$$
\boldsymbol{P}\left\{\Phi_{\omega}(A)=\infty\right\}=m(E)=1,
$$

and the proof of (ii) is complete.

\section{References}

1. M. S. Bartlett, Spectral analysis of point processes, J. Roy. Statist. Soc. Ser. B. 25 (1963), 264-296.

2. V. E. Benes, General Stochastic Processes in the Theory of Queues, Addison-Wesley, 1963.

3. J. L. Doob, Stochastic Processes, Wiley, 1953.

4. J. Feldman, Subinvariant measures for Markoff operators, Duke Math. J. 29 (1962), 71-98.

5. P. R. Halmos, Measure Theory, van Nostrand, 1950.

6. D. G. Kendall, Extreme-point methods in stochastic analysis, Z. Wahrscheinlichkeitstheorie 1 (1963), 295-300.

7. J. F. C. Kingman, The stochastic theory of regenerative events, Z. Wahrscheinlichkeitstheorie 2 (1964), 180-224.

8. P. Lévy, Complément à l'étude des processus de Markoff, Ann. Sci. École Norm. Sup. III 69 (1951), 203-212.

9. M. Loève, Probability Theory, van Nostrand, 1963.

10. C. Ryll-Nardzewski, Remarks on processes of calls, Proc. 4th Berkeley Sympos. Math. Statist. and Prob. 1961, II, 455-465.

11. P. Whittle, On the variation of yield variance with plot size, Biometrika 43 (1956), 337-343.

Received February 24, 1966.

Universities of Cambridge and Sussex, England 


\section{PACIFIC JOURNAL OF MATHEMATICS}

\section{EDITORS}

H. SAMELSON

Stanford University

Stanford, California

J. P. JANS

University of Washington

Seattle, Washington 98105
J. DugundJI

University of Southern California

Los Angeles, California 90007

RICHARD ARENS

University of California

Los Angeles, California 90024

\section{ASSOCIATE EDITORS}

E. F. BECKENBACH

B. H. NeumanN

F WolF

K. YOSIDA

\section{SUPPORTING INSTITUTIONS}

UNIVERSITY OF BRITISH COLUMBIA

CALIFORNIA INSTITUTE OF TECHNOLOGY

UNIVERSITY OF CALIFORNIA

MONTANA STATE UNIVERSITY

UNIVERSITY OF NEVADA

NEW MEXICO STATE UNIVERSITY

OREGON STATE UNIVERSITY

UNIVERSITY OF OREGON

OSAKA UNIVERSITY

UNIVERSITY OF SOUTHERN CALIFORNIA
STANFORD UNIVERSITY

UNIVERSITY OF TOKYO

UNIVERSITY OF UTAH

WASHINGTON STATE UNIVERSITY

UNIVERSITY OF WASHINGTON

AMERICAN MATHEMATICAL SOCIETY CHEVRON RESEARCH CORPORATION TRW SYSTEMS

NAVAL ORDNANCE TEST STATION

Printed in Japan by International Academic Printing Co., Ltd., Tokyo Japan 


\section{Pacific Journal of Mathematics}

\section{Vol. 21, No. $1 \quad$ November, 1967}

Friedrich-Wilhelm Bauer, Der Hurewicz-Satz................... 1

D. W. Dubois, A note on David Harrison's theory of preprimes . ......... 15

Bert E. Fristedt, Sample function behavior of increasing processes with stationary, independent increments ..................... 21

Minoru Hasegawa, On the convergence of resolvents of operators....... 35

Søren Glud Johansen, The descriptive approach to the derivative of a set function with respect to a $\sigma$-lattice ....................... 49

John Frank Charles Kingman, Completely random measures ............ 59

Tilla Weinstein, Surfaces harmonically immersed in $E^{3} \ldots \ldots \ldots \ldots \ldots . . \ldots 9$

Hikosaburo Komatsu, Fractional powers of operators. II. Interpolation spaces ......................................... 89

Edward Milton Landesman, Hilbert-space methods in elliptic partial differential equations ...................................... 113

O. Carruth McGehee, Certain isomorphisms between quotients of a group algebra ........................................ 133

DeWayne Stanley Nymann, Dedekind groups .................. 153

Sidney Charles Port, Hitting times for transient stable processes ......... 161

Ralph Tyrrell Rockafellar, Duality and stability in extremum problems involving convex functions . ............................ 167

Philip C. Tonne, Power-series and Hausdorff matrices . . .............. 189 